\title{
SOFTLY BROKEN SUPERSYMMETRY AND SU(5)
}

\author{
Savas DIMOPOULOS ${ }^{1,2}$ \\ Institute of Theoretical Physics, Stanford University, \\ Institute of Theoretical Physics, University of California, Santa Barbara \\ and \\ University of Michigan, Ann Arbor, USA \\ Howard GEORGI ${ }^{3}$ \\ Lyman Laboratory of Physics, Harvard University, Cambridge, MA 02138, USA
}

Received 2 June 1981

\begin{abstract}
We construct an explicit realistic SU(5) model in which softly broken supersymmetry is used to protect the Higgs doublets from quadratic mass renormalization. The model requires one natural but incredibly accurate adjustment of parameters. We argue that such an adjustment will be required in any supersymmetric GUT in which baryon number is not conserved.
\end{abstract}

\section{Introduction}

In this paper we construct a realistic grand unified theory in which supersymmetry is softly broken at a mass scale of order $1 \mathrm{TeV}$. The light quarks and leptons in the model get mass as in the standard model through their Yukawa couplings to elementary Higgs mesons. The purpose of the softly broken supersymmetry is to protect the scalar mesons from quadratic mass renormalizations which would prevent (or make unnatural) their appearance in the physics below the unification mass scale. The soft breaking of the supersymmetry enables us to build a very straightforward model in which no couplings get large and the masses of all light particles and of their supersymmetric partners are reliably calculated in the tree approximation in the effective low energy theory.

In sect. 2 of this paper we discuss some preliminary concepts which will be important in motivating the specific model. We first discuss the form of the Higgs

\footnotetext{
' Address after January 1, 1982: Lyman Laboratory of Physics, Harvard University, Cambridge, MA 02138 .

${ }^{2}$ This research is supported in part by the National Science Foundation under grant no. PHY77-27084

${ }^{3}$ This research is supported in part by the National Science Foundation under grant no. PHY77-22864.
} 
couplings in a supersymmetric theory. We conclude that two Higgs doublet supermultiplets are required over and above the supermultiplets which contain the quarks and leptons. We then review the subject of coupling constant renormalization in supersymmetric theories. We note that if all couplings are to remain small at the unification scale, $M_{\mathrm{u}}$, the number of supermultiplets which survive below $M_{\mathrm{u}}$ is tightly constrained. In particular, no more than five generations of quarks and leptons are allowed.

In sect. 3 we review the general form of supersymmetric Yang-Mills theories. Then in sect. 4 we describe a specific model which illustrates the physics at the unification scale in theories of this kind. In particular, we describe how to break SU(5) down to $\mathrm{SU}(3) \times \mathrm{SU}(2) \times \mathrm{U}(1)$ while preserving the supersymmetry. At this point, a judicious choice of the parameters in the theory is required to maintain the masslessness of the Higgs doublets while giving large mass to their colored SU(5) partners. We will argue that the required choice, while not particularly attractive, is perfectly natural. Further, we note that any model of this kind requires a similar fine tuning.

We note in passing that this particular supersymmetric theory has several physically inequivalent but degenerate vacua.

The model discussed in sect. 4 is not the end of the story-two more things are required to get a fully realistic model. The supersymmetry must be broken and the Higgs structure must be modified. The model of sect. 4 is chosen for simplicity and pedagogical clarity.

In sect. 5 we discuss supersymmetry breaking. We first state a theorem which implies that spontaneously broken supersymmetry in an SU(5) theory can never give rise to a phenomenologically acceptable mass matrix in tree approximation. There is always a light color triplet boson. After commenting on the possibility that acceptable supersymmetry breaking mass splitting may arise as a radiative correction in a "supercolor" theory, we proceed to a discussion of soft supersymmetry breakdown. We find that fermion-boson mass splittings of the order of $1 \mathrm{TeV}$ can be put in by hand without inducing large quadratic renormalizations of the Higgs mass. Then the two mass scales in the theory are associated with very different physics. The unification mass is the scale set by the supersymmetric interactions. The $S U(2) \times U(1)$ breaking scale is associated with the soft supersymmetry breaking.

In sect. 6 we discuss the phenomenology of the resulting theory. It is very sparse. All the supersymmetric partners of the usual fields are heavy. The lightest one is stable. One interesting feature is that flavor-changing effects from loops involving the supersymmetric partners are suppressed by a super-GIM mechanism.

We next discuss the lepton-quark mass relations that arise from the simple model of sect. 4. We find that we must complicate the Higgs structure of the model, but the qualitative structure remains unchanged.

Sect. 7 contains conclusions. In appendix A we discuss the mass matrix in a general supersymmetric theory in a useful notation. In appendix $B$, we prove the theorem stated in sect. 4 . 


\section{Matter versus Higgs}

In any theory in which supersymmetry is relevant down to $1 \mathrm{TeV}^{\star}$, the supersymmetric partners of the leptons are spinless bosons with the right $S U(2) \times U(1)$ properties to be Higgs mesons. However, for various reasons, they cannot be. The most serious objection to using the lepton partners as Higgs mesons is that the partners of the charge $-\frac{1}{3}$ quarks then mediate baryon number changing interactions. To eliminate this difficulty, the Higgs doublets must be part of a different SU(5) multiplet whose color triplet components are superheavy. To ensure that baryon number is conserved except by the usual SU(5) baryon number changing interactions, we require that the Higgs multiplets couple to pairs of quark and lepton multiplets just as in the usual SU(5) model.

We will call the quark and lepton supermultiplets matter supermultiplets. The above constraint can be restated by saying that the matter supermultiplets always couple in pairs to the Higgs supermultiplets.

In a supersymmetric theory, the supermultiplets carry a handedness, that of the fermions they contain. Only supermultiplets of the same handedness can have Yukawa couplings to one another. Thus the Higgs which give mass to charge $\frac{2}{3}$ quarks and those which give mass to the charge $-\frac{1}{3}$ quarks cannot be charge conjugates of one another-the handedness of associated fermions must be the same. We need two left-handed Higgs doublet supermultiplets, one with average charge $\frac{1}{2}$ which gives mass to the charge $\frac{2}{3}$ quarks and one with average charge $-\frac{1}{2}$ which gives mass to the charge $-\frac{1}{3}$ quarks.

We next review the subject of coupling constant renormalization in a supersymmetric SU(5) theory. The basic difference between supersymmetric SU(5) and the standard SU(5) theory is that the supersymmetric partners of the light gauge bosons are not superheavy. This makes the $\mathrm{SU}(3), \mathrm{SU}(2)$, and $\mathrm{U}(1)$ gauge couplings approach one another more slowly than in the usual theory, so the unification scale is increased [2]. This doesn't affect the prediction of the weak mixing angle. But the presence of extra light Higgs doublets does tend to increase $\sin ^{2} \theta$, as we will see quantitatively below. We will also want to keep track of the number of matter multiplets to check that the gauge couplings do not get large before unification.

Call the number of Higgs doublet supermultiplets $h$ and the number of matter doublet supermultiplets $m$ (there are four doublets for each generation of quarks and leptons, so $m=12$ for three generations). Then the gauge couplings at $M_{\mathrm{w}}$ satisfy

$$
\begin{gathered}
\frac{2 \pi}{\alpha_{\mathrm{s}}}=\frac{2 \pi}{\alpha_{0}}+\left(9-\frac{1}{2} m\right) \ln \frac{M_{\mathrm{W}}}{M_{\mathrm{u}}}, \\
\sin ^{2} \theta \frac{2 \pi}{\alpha}=\frac{2 \pi}{\alpha_{0}}+\left(6-\frac{1}{2} h-\frac{1}{2} m\right) \ln \frac{M_{\mathrm{W}}}{M_{\mathrm{u}}}, \\
\frac{3}{5} \cos ^{2} \theta \frac{2 \pi}{\alpha}=\frac{2 \pi}{\alpha_{0}}+\left(-\frac{3}{10} h-\frac{1}{2} m\right) \ln \frac{M_{\mathrm{W}}}{M_{\mathrm{u}}},
\end{gathered}
$$

\footnotetext{
* For a review of supersymmetry and references to the literature, see ref. [1].
} 
where $\alpha_{0}$ is the gauge coupling at unification and $M_{\mathrm{u}}$ is the mass of the leptoquark gauge supermultiplets. Solving (2.1), we find

$$
\begin{aligned}
\frac{1}{\alpha_{0}} & =\frac{\left(9-\frac{1}{2} m\right) / \alpha-\left(6-h-\frac{4}{3} m\right) / \alpha_{\mathrm{s}}}{18+h}, \\
\ln \frac{M_{\mathrm{u}}}{M_{\mathrm{w}}} & =\frac{2 \pi / \alpha-16 \pi / 3 \alpha_{\mathrm{s}}}{18+h} \\
\sin ^{2} \theta & =\frac{\left(3+\frac{1}{2} h\right)+\left(10-\frac{1}{3} h\right) \alpha / \alpha_{\mathrm{s}}}{18+h} .
\end{aligned}
$$

Inserting a reasonable value for the ratio $\alpha / \alpha_{\mathrm{s}}$ at $M_{\mathrm{W}}, \alpha / \alpha_{\mathrm{s}}=5.57 \times 10^{-2}$ (corresponding roughly to $\Lambda=0.3$ ), we find for $h=2$

$$
\begin{aligned}
\sin ^{2} \theta & =0.226, \\
M_{\mathrm{u}} & \simeq 10^{17} \mathrm{GeV} .
\end{aligned}
$$

These results are independent of $m$, as usual in a one-loop calculation. But as (2.2) shows, $m$ is highly constrained by the constraint of perturbative unification, the requirement that $\alpha_{0}$ be small. $\alpha_{0}$ is of order one if $m=20$. Thus at most five generations are allowed by perturbative unification.

\section{Notation}

A general supersymmetric Yang-Mills theory is characterized by specifying the gauge group and gauge coupling constants, the representation under which all the left-handed supermultiplets transform, and a single gauge invariant function $v$ of the left-handed fields. The following notation is very convenient ${ }^{\star}$ : Let $\psi^{a}$ ( $A=1$ to $N$ ) be the left-handed fermion fields associated with the scalar supermultiplets and $\phi^{a}$ be the corresponding complex spinless meson fields. Let $A_{\alpha}^{\mu}$ be the gauge fields (where $\mu$ is a vector index and $\alpha$ labels the generators of the gauge group) and $\chi_{\alpha}$ be their supersymmetric partners which we can take to be left-handed fermion fields. The covariant derivative on the $\psi$ and $\phi$ fields is

$$
D_{b}^{\mu a}=\partial^{\mu} \delta_{b}^{a}+i g_{\alpha} A_{\alpha}^{\mu} T_{\alpha b}^{a},
$$

where $T_{\alpha}$ is the gauge generator and $g_{\alpha}$ is the gauge coupling constant. The

\footnotetext{
* The notation here is similar in spirit to that of ref. [3].
} 
gauge-invariant function is $v(\phi)$. We will also need the derivatives

$$
\begin{gathered}
v_{a}(\phi)=\frac{\partial}{\partial \phi^{a}} v(\phi), \\
v_{a b}(\phi)=\frac{\partial^{2}}{\partial \phi^{a} \partial \phi^{b}} v(\phi), \\
v_{a b c}(\phi)=\frac{\partial^{3}}{\partial \phi^{a} \partial \phi^{b} \partial \phi^{c}} v(\phi) .
\end{gathered}
$$

Gauge invariances of $v$ implies

$$
v_{a}(\phi) T_{b}^{a} \phi^{b}=0
$$

(summation over repeated indices implied). The penultimate piece of notation we need is for the complex conjugates of $\phi^{a}$ :

$$
\bar{\phi}_{a}=\phi^{a *}, \quad \bar{v}(\bar{\phi})=v(\phi)^{*}, \quad \bar{v}^{a}(\bar{\phi})=v_{a}(\phi)^{*},
$$

etc. Finally, we define the functions

$$
K_{\alpha}=g_{\alpha} \bar{\phi}_{a} T_{\alpha b}^{a} \phi^{b}
$$

(no sum on $\alpha$ ) with derivatives defined as in (3.2) and (3.4).

To put all this together into the lagrangian for the supersymmetric theory, it is convenient to work in a Majorana basis for the fermion fields. The kinetic energy terms and the gauge couplings are the usual ones with the covariant derivatives given by (3.1). The Yukawa couplings and fermion mass terms are

$$
-\frac{1}{2} \psi^{a \mathrm{~T}} \gamma^{0} v_{a b}(\phi) \psi^{b}+\text { h.c. }, \quad-\chi_{\alpha} \gamma^{0} K_{\alpha a}(\bar{\phi}) \psi^{a}+\text { h.c. }
$$

The scalar meson potential is

$$
v(\phi, \bar{\phi})=v_{a}(\phi) \bar{v}^{a}(\bar{\phi})+\frac{1}{4} K_{\alpha} K_{\alpha}
$$

The vacuum expectation value (VEV) of the $\phi$ 's is determined by minimizing (3.7). In most cases, the minimum will occur when

$$
v_{a}(\phi)=\bar{v}^{a}(\bar{\phi})=K_{\alpha}=0
$$

If (3.8) can be satisfied, then although the gauge symmetries may be spontaneously broken, the supersymmetry remains unbroken. It is sometimes possible to arrange 
$v(\phi)$ so that (3.8) is not allowed, so that (3.7) has its minimum for nonzero values of $v_{a}(\phi)$ and $K_{\alpha}$. In this case the supersymmetry is broken spontaneously. We will discuss spontaneously broken supersymmetry further in appendix A. We will not pursue it further here because, as we shall see, it does not lead to a sensible phenomenology in tree approximation.

From (3.6), (3.7) it is clear that if the theory is to be renormalizable by conventional power counting, $v(\phi)$ can be at most cubic in the fields. We will assume that this constraint is satisfied.

If this were an ordinary field theory, we would expect quantum loop corrections to renormalize the parameters in $v(\phi)$ and to generate effective (finite) nonrenormalizable terms in $v$. The weird, astonishing, unique thing about supersymmetric theories is that these things do not happen. The parameters in $v(\phi)$ are modified by radiative corrections only through wave-function renormalization [1].

\section{4. $\mathrm{SU}(5)$}

We can now describe a simple SU(5) model which illustrates the first half of our program; the breaking of $S U(5)$ down to $S U(3) \times S U(2) \times U(1)$ preserving supersymmetry and producing a pair of massless Higgs doublets.

As noted in sect. 3 , we can characterize the theory by giving the SU(5) transformation properties of the fields and specifying the SU(5) and invariant function $v$. The left-handed supermultiplets are the following (all of these correspond to the $\phi^{a}$ of sect. 3):

$$
\begin{aligned}
& H^{x}, \quad H_{x}^{\prime}, \\
& M_{j}^{x y}=-M_{j}^{y x}, \quad M_{j x}^{\prime}, \\
& \Sigma_{y}^{x} .
\end{aligned}
$$

The $x$ and $y$ are SU(5) indices and $j$ is a flavor index. $H^{x}$ is a 5 and $H_{x}^{\prime}$ is a $\overline{5}$. The SU(2) doublet components of these are the Higgs supermultiplets. $M_{j}^{x y}$ are 10 's and $M_{j x}$ are $\overline{5}$ 's. These are the matter supermultiplets. $\Sigma_{y}^{x}$ is a 24 whose VEV will break SU(5).

The function $v$ is given by

$$
\begin{gathered}
\lambda_{1}\left(\frac{1}{3} \Sigma_{y}^{x} \Sigma_{z}^{y} \Sigma_{x}^{z}+\frac{1}{2} m \Sigma_{y}^{x} \Sigma_{x}^{y}\right)+\lambda_{2} H_{x}^{\prime}\left(\Sigma_{y}^{x}+3 m^{\prime} \delta_{y}^{x}\right) H^{y} \\
+f_{j k} \varepsilon_{u v w x y} H^{u} M_{j}^{v w} M_{k}^{x y}+g_{j k} H_{x}^{\prime} M_{j}^{x y} M_{k y}^{\prime} .
\end{gathered}
$$

We can now apply the formalism of sect. 3 to (4.2) with the following results: the 
potential is minimized for

$$
\left\langle H^{x}\right\rangle=\left\langle H_{x}^{\prime}\right\rangle=\left\langle M_{j}^{x y}\right\rangle=\left\langle M_{j x}\right\rangle=0
$$

and one of three VEV's for $\Sigma$ :

$$
\begin{aligned}
& \left\langle\Sigma_{y}^{x}\right\rangle=0 \\
& \left\langle\Sigma_{y}^{x}\right\rangle=\frac{1}{3} m \delta_{y}^{x}-\frac{5}{3} m \delta_{5}^{x} \delta_{y}^{5}, \\
& \left\langle\Sigma_{y}^{x}\right\rangle=2 m \delta_{y}^{x}-5 m\left(\delta_{4}^{x} \delta_{y}^{4}+\delta_{5}^{x} \delta_{y}^{5}\right) .
\end{aligned}
$$

Supersymmetry is not broken. (4.4a) does not break SU(5). (4.4b) breaks it down to $\mathrm{SU}(4) \times U(1) .(4.4 c)$ breaks it down to $\mathrm{SU}(3) \times \mathrm{SU}(2) \times \mathrm{U}(1)$. At this point, these three physically inequivalent vacua are completely degenerate. This bizarre situation is not uncommon in supersymmetric theories (it may be cosmologically relevant!).

In any event, (4.4c) is the solution of interest. For this solution, the $\mathrm{SU}(2)$ doublet Higgs, $H^{4,5}$ and $H_{4,5}^{\prime}$, have a mass squared which is proportional to $m-m^{\prime}$. If we set $m^{\prime}$ exactly equal to $m$, they are massless, while the color triplet components $H^{1,2,3}$ and $H_{1,2,3}^{\prime}$ have mass of order $m$. This is the result we want, so we require in (4.2)

$$
m^{\prime}=m=\mathrm{O}\left(M_{\mathrm{u}}\right)
$$

It may seem that we have accomplished nothing by introducing supersymmetry. We are still required to make an incredibly accurate adjustment of the parameters in our theory. But there is at least a technical difference between (4.5) and the adjustments we have to make in a non-supersymmetric theory. (4.5) need only be imposed once, at the very beginning, because the ratio $\mathrm{m}^{\prime} / \mathrm{m}$ is not renormalized at all. Both $m^{\prime}$ and $m$ are renormalized like the $\Sigma$ field. Thus the imposition of (4.5) is natural in a technical sense ${ }^{\star}$.

At momenta small compared to $m$, the theory collapses into an effective $\mathrm{SU}(3) \times$ $\mathrm{SU}(2) \times U(1)$ supersymmetric gauge theory, in which the only relevant fields are the matter supermultiplets (the debris of $M_{j}^{x y}$ and $M_{j x}$ ), the $\mathrm{SU}(3) \times \mathrm{SU}(2) \times \mathrm{U}(1)$ gauge supermultiplets, and the two massless Higgs supermultiplets. The matter fields are massless because they are protected by an unbroken chiral gauge symmetry of the full theory. The gauge symmetry also protects the gauge fields. The Higgs fields are massless because we made them massless. It is true that in the effective low-energy theory there is a global chiral symmetry that protects the Higgs fields. But as the above analysis shows, it cannot be a symmetry of the full theory. It is broken by the interactions which give large mass to the color triplet relatives of the Higgs doublets. * This is naturalness in the sense of no infinite renormalization, as in ref. [4a], rather than in the sense
of ref. [4b]. 
If we did not have to make the color triplets heavy, we could take $\lambda_{2}=0$. Then both the doublets and the triplets would be massless in the supersymmetric theory. They would be protected by a global chiral symmetry of the full theory. In a standard SU(5) theory, the triplets must be very heavy because they mediate baryon number violating processes. We expect a similar situation in any unified theory in which the Higgs doublets are unified with fields that can produce baryon number changing effects. It is conceivable that in a theory with global baryon number conservation the entire multiplet including the Higgs doublets could be kept massless by a global symmetry, without any adjustments at all.

At this point, except for the multiplicity of Higgs, we have a simple doubling of the degrees of freedom. Degenerate with the twelve SU(3), SU(2), and U(1) gauge bosons are twelve massless gauge fermions. The matter fermions are accompanied by matter bosons with the same quantum numbers, and the Higgs bosons with Higgs fermions.

One might worry that the matter bosons would have large baryon number violating self-interactions because a four-boson term, unlike a four-fermion term, is not necessarily suppressed by powers of $M_{\mathrm{u}}^{-1}$. In particular, the terms in the potential of the form $K_{\alpha} K_{\alpha}$, where $\alpha$ runs over the leptoquark gauge bosons in $\mathrm{SU}(5)$, contain baryon number violating four-matter-boson interaction terms. But the contribution of these terms is cancelled by a similar effect from the coupling (again in the $K_{\alpha} K_{\alpha}$ term) of the matter bosons to the massive boson in the massive vector supermultiplet with the $A_{\alpha}^{\mu}$-field. Indeed, the $K_{\alpha} K_{\alpha}$ term is essentially just a mass term for this massive boson, and it leaves no trace in the effective low-energy theory. This, of course, is what we expected. The supersymmetry of the effective low energy theory should rule out any $K_{\alpha} K_{\alpha}$ terms except those associated with the unbroken $S U(3) \times S U(2) \times U(1)$ gauge symmetries. But it is nice to see the unwanted terms disappearing explicitly.

\section{Soft supersymmetry breaking}

In the simple model of sect. 4, spinless fields do not develop a supersymmetry breaking VEV at any mass scale. However, we could modify the model so that the effective low energy theory would break supersymmetry spontaneously at around a $\mathrm{TeV}$. The question is, do we get a realistic theory with such a strategy? The answer is no, at least not in tree approximation. This can be seen from the following theorem.

Theorem: In an $\mathrm{SU}(3) \times \mathrm{SU}(2) \times \mathrm{U}(1)$ theory of quarks and leptons with spontaneously broken supersymmetry, the tree approximation mass matrix describes a color triplet spinless meson of one of the following kinds; a charge $\frac{2}{3}$ triplet not heavier than the lightest charge $\frac{2}{3}$ quark, or a charge $-\frac{1}{3}$ triplet not heavier than the lightest charge $-\frac{1}{3}$ quark. This theorem is proven rigorously in appendix B. Loosely speaking, it is true because spontaneous supersymmetry breaking leaves the fermion mass matrix unchanged while it splits the spinless mesons in opposite directions. 
Thus if we break supersymmetry spontaneously, we must rely on radiative corrections to give a large mass to the supersymmetric partners of the quarks. This is only reasonable if some of the couplings get large, as in a supercolor theory [5].

Here we explore a simpler alternative possibility-that the supersymmetry is broken explicitly, but softly, by terms of dimension less than four in the lagrangian. We add to the lagrangian (not to $v$ ) the following SU(5) invariant mass terms, all of the order of a TeV:

(1) a positive mass squared term for the matter bosons;

(2) a mass for the Higgs fermions (and their SU(5) partners);

(3) a Majorana mass for the gauge fermions;

(4) a negative mass squared term for the boson fields in the $\Sigma$ supermultiplet;

(5) a mixed (with positive and negative eigenvalues) mass squared matrix for the Higgs bosons.

The effect of (1)-(3) is simply to increase the mass of unobserved particles which would otherwise be light or massless. (4) doesn't do much to the low-energy theory, but it does pick out the $\mathrm{SU}(3) \times \mathrm{SU}(2) \times \mathrm{U}(1)$ symmetry breaking as the lowest energy state of the full theory. (5) produces a non-zero VEV for the Higgs bosons. The form of the Higgs boson mass matrix must be chosen with care to assure that the hamiltonian is bounded below because the Higgs boson potential in the effective low energy theory is flat in some directions. Also, the supersymmetric low energy lagrangian has a Peccei-Quinn symmetry which can be broken by the explicit Higgs boson mass squared term to eliminate the axion.

We see no essential difference between the soft breaking of supersymmetry and the soft breaking of an ordinary symmetry. The Symanzik theorem ought to apply to either case [6].

Grisaru has given an elegant argument that a scalar meson mass term is a consistent soft breaking by showing that it is equivalent to a coupling to an external vector supermultiplet with a constant auxiliary field component [7]. We know of no similar argument for a fermion mass term, but we can think of no reason why it should not make sense.

It is amusing that the two mass scales in this theory arise from completely different physics. The unification scale, $M_{\mathrm{u}}$, is essentially the mass parameter in the supersymmetric theory. The $\mathrm{SU}(2) \times \mathrm{U}(1)$ scale, $M_{\mathrm{W}}$, is determined by the scale of the explicit soft supersymmetry breaking.

\section{Physics}

The phenomenology of this model is simple. In addition to the usual light matter fermions, gauge bosons and Higgs bosons, we predict heavy matter bosons, gauge fermions and Higgs fermions as supersymmetric partners. We can say little about their masses except that they cannot be very large compared to $1 \mathrm{TeV}$ or the motivation for the model disappears. 
The lightest of the supersymmetric partners is stable. The others all decay into it plus ordinary particles. One simple possibility is to imagine that the lightest particle is the $\mathrm{U}(1)$ gauge fermion. In this respect, the model differs somewhat from a supercolor model with spontaneously broken supersymmetry in which all the supersymmetric particles can decay into their ordinary partners plus a Goldstone fermion.

One interesting sidelight is the mechanism by which the model avoids large flavor changing effects from loops involving the heavy particles. A potentially dangerous diagram is the $\Delta S=2$ superbox diagram of fig. 1. So long as we are careful to break the supersymmetry by adding a common mass to all the matter bosons with the same quantum numbers, this diagram is suppressed by a super-GIM mechanism. Like the usual box diagram, it is proportional to $\left(m_{\mathrm{c}}^{2}-m_{\mathrm{u}}^{2}\right)^{2}$ (or similar differences involving the t-quark mass) because the mass squared differences of the quarks are the same as those of their supersymmetric partner matter bosons. In fact this diagram is completely negligible.

In one respect, the simple model of sect. 3 may be inadequate to describe the physics we see. As in any SU(5) theory in which the Higgs mesons transform as part of a (5), the charge $-\frac{1}{3}$ quark and charged lepton masses are equal at the unification scale. This leads to a prediction [8] for the $b / \tau$ mass ratio which (remarkably) is similar to that in the standard SU(5) model. But it also leads to the (possibly) bad relation $\mathrm{b} / \tau=\mathrm{s} / \mu=\mathrm{d} / \mathrm{e}$.

If this difficulty is real, then the light Higgs which gives mass to the charge $-\frac{1}{3}$ quarks and charged leptons must be a linear combination of the doublets in a $\overline{5}$ and $\overline{45}$. But they probably cannot both be light. That would give an unacceptably large contribution to $\sin ^{2} \theta$. We can, however, construct the theory so that the Higgs doublet which is light is a linear combination of the doublet components of the $\overline{5}$ and $\overline{45}$. The orthogonal component would be superheavy and not get any VEV. Again, this can be arranged. Although the theory is somewhat cumbersome, the idea is exactly the same as in the model of sect. 4 . The fine adjustments required are all natural.

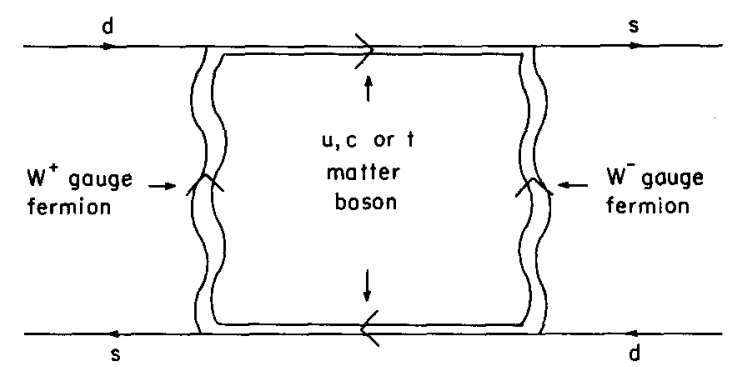

Fig. 1. The $\Delta S=2$ superbox diagram. The virtual particles are the supersymmetric partners of those in the ordinary box diagram. 


\section{Conclusions}

We have shown that supersymmetry can be incorporated into a unified theory in an interesting way which makes use of the special renormalization properties of supersymmetric theories. We have stressed that while we can use supersymmetry to make the Higgs doublets massless, the masslessness is not automatic or trivial. It requires an exact but natural adjustment of parameters.

In discussing the masslessness of the Higgs doublet in a grand unified model, Weinberg said with some justice that the required adjustment of parameters was unnatural but not unattractive [9]. With even more justice, we can say that the adjustment we must make in this theory is unattractive but not unnatural.

In the specific model we discuss, the $\mathrm{SU}(2) \times \mathrm{U}(1)$ breaking scale is introduced through the explicit supersymmetry breaking. It may be possible to introduce this scale dynamically through spontaneous symmetry breaking in a supercolor model or perhaps in some other way [1]. We hope that the explicit breaking scheme we have discussed will be useful, at least as an illustration of how easy it is to use supersymmetry in a realistic unified model.

We would like to thank L. Girardello and M. Grisaru for extremely helpful discussions of supersymmetry. We are particularly grateful for their patience in explaining superfield perturbation theory and the non-renormalization theorems (in spite of our initial skepticism) and for their help in understanding soft supersymmetry breakdown. One of us (HG) would like to acknowledge countless useful discussions with an old officemate, E. Witten.

\section{Note added in proof}

The notation of sect. 3 is correct for an unconventional normalization of the scalar fields. The standard normalization is obtained by multiplying $K$ (and its derivatives) by $\sqrt{ } 2$ wherever it appears. We thank $S$. Weinberg for pointing this out. Weinberg also found an interesting source of baryon number violation in our model which arises only in one loop but is suppressed by only two powers of $M_{\mathrm{u}}$ (in the rate).

One of us (HG) thanks Einhorn and Jones [8] for pointing out an error in his calculation of the $b / \tau$ mass ratio.

A model virtually identical to ours has been constructed independently by $\mathrm{N}$. Sakai (Tohoku Univeristy preprint TU/81/225).

\section{Appendix A}

Here we expand on the notation of sect. 3 and write down the mass matrices. These depend only on the VEV's of the functions $v(\phi), v_{a}(\phi) \cdots$ and $K_{\alpha}(\bar{\phi}, \phi)$, $K^{a}(\phi) \cdots$. We will denote the VEV's by the same symbols without explicit $\phi$ dependence. In this notation, the condition that the VEV extremizes the potential is

$$
v_{a b} \vec{v}^{b}+\frac{1}{2} K_{\alpha a} K_{a}=0 .
$$


The mass squared matrix of the vector bosons is given by

$$
\frac{1}{2}\left(K_{\alpha a} K_{\beta}^{a}+K_{\alpha}^{a} K_{\beta a}\right) .
$$

The mass matrix of the left-handed fermions is the symmetric matrix

$$
\left[\begin{array}{cc}
v_{a b} & K_{\alpha b} \\
K_{\alpha a} & 0
\end{array}\right]
$$

The mass squared matrix of the spinless bosons is

$$
\left[\begin{array}{ll}
\bar{v}^{a b} v_{b c}+\frac{1}{2} K_{\alpha}^{a} K_{\alpha c}+\frac{1}{2} K_{\alpha c}^{a} K_{\alpha} & \bar{v}^{a b c} v_{b}+\frac{1}{2} K_{\alpha}^{a} K_{\alpha}^{c} \\
v_{a b c} v^{b}+\frac{1}{2} K_{\alpha a} K_{\alpha c} & v_{a b} \bar{v}^{b c}+\frac{1}{2} K_{\alpha a} K_{\alpha}^{c}+\frac{1}{2} K_{\alpha a}^{c} K_{\alpha}
\end{array}\right] .
$$

The signal of spontaneous supersymmetry breakdown is a non-zero value of $v_{a}$ or $K_{\alpha}$. If one or both of these is non-zero, there is a Goldstone fermion, in the direction of the vector

$$
\left[\begin{array}{c}
v_{a} \\
\frac{1}{2} K_{\alpha}
\end{array}\right]
$$

It is easy to check that this annihilates (A.3), using (A.1) and (3.3) which is equivalent to

$$
v_{a} K_{\alpha}^{a}=0
$$

Note that if $v_{a}=K_{\alpha}=0$, then

$$
\begin{aligned}
v^{a b} K_{\alpha b} & =0, \\
K_{\alpha a} K_{\beta}^{a} & =K_{\beta a} K_{\alpha}^{a} .
\end{aligned}
$$

With these relations you can easily show that in the supersymmetric limit, the theory falls apart in the correct way into degenerate supermultiplets.

\section{Appendix B}

Here we prove the theorem stated in sect. 5 . The reason that we look specifically at the quarks, rather than the leptons, is that in the effective low-energy SU(3) $\times$ $\mathrm{SU}(2) \times \mathrm{U}(1)$ gauge theory there are no color triplet gauge bosons. Therefore, the $K_{\alpha a}$ and $K_{\alpha}^{a}$ terms vanish by SU(3) symmetry. Then we can write the mass matrices in the color triplet sector in a very simple form. Consider the charge $\frac{2}{3}$ quarks (the charge $-\frac{1}{3}$ quarks have the same form). If the mass matrix for the quarks is $m$, the mass squared matrix for the color triplet bosons is

$$
M^{2}=\left[\begin{array}{cc}
m m^{\dagger}+g_{\alpha} K_{\alpha} T_{\alpha}^{\mathrm{R}} & \Delta \\
\Delta^{\dagger} & m^{\dagger} m+g_{\alpha} K_{\alpha} T_{\alpha}^{\mathrm{L}}
\end{array}\right],
$$

where $T_{\alpha}^{\mathrm{L}, \mathrm{R}}$ are the gauge generators on the left- and right-handed fields. 
The only $\alpha$ which are relevant are those for the $U(1)$ and the neutral component of SU(2) because only these are color and electromagnetically neutral. The point is that $g_{\alpha} K_{\alpha} T_{\alpha}$ is traceless over all the color triplet fields. Thus at least one of the $g_{\alpha} K_{\alpha} T_{\alpha}^{\mathrm{L} \text { or } \mathrm{R}}$ for charge $\frac{2}{3}$ or charge $-\frac{1}{3}$ fields must be negative or zero. Suppose (for example) it is for the right-handed charge $\frac{2}{3}$ fields. Then if $\xi$ is the normalized eigenvector of $\mathrm{mm}^{\dagger}$ with minimum eigenvalue $\left(\mathrm{m}_{0}^{2}\right)$

$$
m m^{\dagger} \xi=m_{0}^{2} \xi
$$

the vector

$$
Z=\left(\begin{array}{l}
\xi \\
0
\end{array}\right)
$$

satisfies

$$
Z^{\dagger} M^{2} Z \leqslant m_{0}^{2} Z^{\dagger} Z=m_{0}^{2}
$$

Therefore $M^{2}$ has an eigenvalue smaller than $m_{0}^{2}$ and the theorem is proved.

\section{References}

[1] J. Wess and B. Zumino, Nucl. Phys. B70 (1974) 39;

E. Witten, Nucl. Phys. B188 (1981) 513

[2] S. Dimopoulos, S. Raby and F. Wilczek, to be published

[3] S. Ferrara, L. Girardello and F. Palumbo, Phys. Rev. D20 (1979) 403

[4] (a) H. Georgi and A. Pais, Phys. Rev. D10 (1974) 539 and references [2-8] therein

(b) G. 't Hooft, Lecture at Cargése Summer Inst., 1979, in Recent developments in gauge theories, ed.

G. 't Hooft et al. (Plenum, 1980)

[5] S. Dimopoulos and S. Raby, Nucl. Phys. B192 (1981) 353;

M. Dine, W. Fischler and M. Srednicki, Nucl. Phys. B189 (1981) 575

[6] K. Symanzik, in Coral Gables Conf. on Fundamental interactions at high energies II, ed. A. Perlmutter, G.J. Iverson and R.M. Williams (Gordon and Breach, New York, 1970);

S. Coleman, Proc. 1971 Int. School of Physics, "Ettore Majorana" (Academic, New York) to be published

[7] L. Girardello and M. Grisaru, Nucl. Phys., to be published

[8] M.B. Einhorn and D.R.T. Jones, Michigan preprint UMHE81-55 (1981)

[9] S. Weinberg, private communication in discussions of Phys. Lett. 82B (1979) 387 